\title{
Adaptive optics in microscopy
}

\author{
By Martin J. Booth* \\ Department of Engineering Science, University of Oxford, \\ Parks Road, Oxford OX1 3PJ, UK
}

The imaging properties of optical microscopes are often compromised by aberrations that reduce image resolution and contrast. Adaptive optics technology has been employed in various systems to correct these aberrations and restore performance. This has required various departures from the traditional adaptive optics schemes that are used in astronomy. This review discusses the sources of aberrations, their effects and their correction with adaptive optics, particularly in confocal and two-photon microscopes. Different methods of wavefront sensing, indirect aberration measurement and aberration correction devices are discussed. Applications of adaptive optics in the related areas of optical data storage, optical tweezers and micro/nanofabrication are also reviewed.

Keywords: adaptive optics; aberrations; confocal microscopy; multiphoton microscopy; optical data storage; optical tweezers

\section{Introduction}

Optical microscopes are essential tools in many scientific fields. In the life sciences, they are widely used for the visualization of cellular structures and subcellular processes. Confocal and multiphoton microscopes are particularly important in this respect as they produce three-dimensional images of volumetric objects. However, the resolution of these microscopes is often adversely affected by the optical properties of the specimen itself. Spatial variations in the refractive index of the specimen introduce optical aberrations that compromise image quality. This is a particular problem when imaging deep into thick biological specimens. Ultimately, the aberrations restrict the amount of specimen that can be observed by the microscope, the depth being limited to a few cellular layers near the surface. This is a serious limitation if one wants to observe cells and their processes in their usual environment, rather than in the unnatural surroundings of a microscope slide.

Until a few years ago, this was a problem that many people knew about, but nobody could fix. Recently, researchers have started to use adaptive optics, a technique borrowed from astronomy, to measure and correct the aberrations and restore the optimum resolution of these systems. The techniques were originally developed for astronomical and military purposes, for stabilizing and de-blurring telescope images of stars and satellites affected by the aberrations caused by atmospheric turbulence (Tyson 1991; Hardy 1998). Adaptive optics technology is

*martin.booth@eng.ox.ac.uk

One contribution of 20 to a Triennial Issue 'Chemistry and engineering'. 
now being developed for more down-to-earth reasons, in smaller scale applications such as ophthalmic imaging and communications. Naturally, the same principles can be applied to microscopy.

The most widely used optical microscopes for high-resolution biomedical imaging are the scanning confocal and two-photon fluorescence microscopes (Wilson 1990; Gu 1996; Pawley 2006). One of the major strengths of these methods is their ability to provide three-dimensional images of volumetric specimens. In the confocal microscope, the three-dimensional resolution arises through the use of a pinhole situated before the photodetector. This pinhole obscures out-of-focus light and allows only light from the focus to be efficiently detected. The two-photon microscope relies upon the process of two-photon excitation fluorescence, in which a fluorophore is excited by the simultaneous absorption of two photons. As the efficiency of two-photon excitation process is proportional to the square of the illumination intensity, fluorescence is only efficiently generated in the focal spot, where the intensity is highest. The twophoton microscopy therefore exhibits intrinsic three-dimensional resolution without the need for a detector pinhole (Sheppard \& Gu 1990).

Aberrations affect all types of microscopy, but the effects are particularly significant for both confocal and two-photon microscopy, causing a reduction in signal level, contrast and resolution. By correcting the aberrations, adaptive optics can improve the image quality or, in the best case, restore the optimum performance of the microscope. Several such adaptive optics systems have been implemented in microscopes and other closely related optical engineering systems. The requirements of these systems have led to the development of several new techniques. In this review, I present an overview of aberrations in microscopy and describe recent developments in microscope adaptive optics.

\section{Aberrations in microscopy}

\section{(a) Description of wavefront aberrations}

A high numerical aperture (NA) objective lens transforms planar wavefronts that pass through the lens pupil into spherical wavefronts in the focal region (figure 1). As the light passes through the optical system and the specimen, aberrations can be introduced that distort the wavefronts from this ideal planar or spherical form. These aberrations can be modelled as phase variations in the pupil of the objective lens. In principle, the aberrations are compensated by introducing an equal but opposite phase aberration into this pupil (or its optical conjugate) using an appropriate correction element. In order to reduce the amount of information required to specify aberrations, it is useful to represent them as an expansion in orthogonal functions. In this way, the aberration can often be accurately represented by a short sequence of function coefficients. In systems with circular pupils, the Zernike circle functions are regularly used as, in addition to their convenient mathematical properties, the low-order Zernike modes correspond closely to traditional aberration terms, such as astigmatism, coma or spherical aberration (Zernike 1934; Born \& Wolf 1983; Mahajan 2001). Other sets of modes may also be appropriate, such as the eigenmodes of the correction element (Paterson et al. 2000). 
(a)

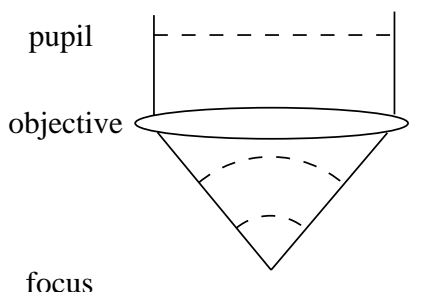

(b)

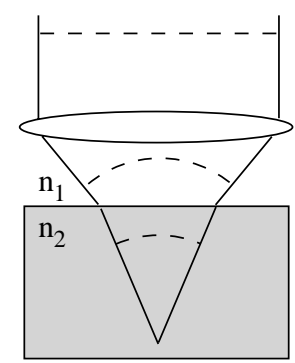

(c)

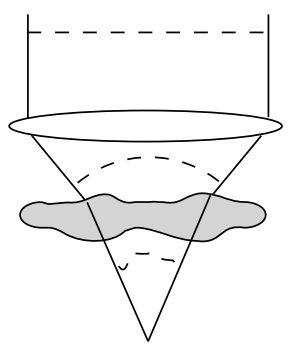

(d)

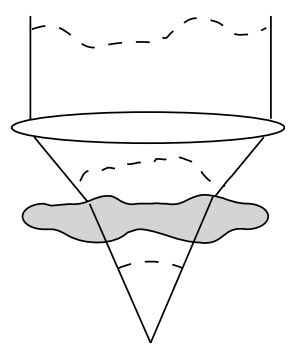

Figure 1. (a) Schematic of focusing by a high-NA objective lens. Planar wavefronts in the pupil are converted into convergent spherical wavefronts in the focus. (b) The effects of focusing through a refractive index mismatch, where refraction at the interface distorts the wavefronts. $(c)$ Focusing through a complex specimen, where refractive index variations introduce aberrations. $(d)$ The principle of aberration correction - a conjugate phase introduced in the pupil is cancelled out by the specimen-induced aberrations.

\section{(b) Sources of aberrations}

One of the most studied specimen geometries is that of the planar refractive index mismatch, where the light is focused through a planar interface between two media with different refractive indices (figure $1 b$ ). This structure is common to many applications: it occurs in microscopy when focusing using an immersion objective, through a cover glass, or into a specimen-mounting medium. The effects of such refractive index mismatch on focusing were first investigated by Sheppard and colleagues (Sheppard \& Cogswell 1991; Sheppard \& Gu 1991). A comprehensive review of further studies pre-1998 is provided by Egner \& Hell (1998). The primary effects of the refractive index mismatch are focal shift and spherical aberration that are proportional to the focusing depth; these can jointly be represented by a series of rotationally symmetric Zernike modes (Török et al. 1995; Booth et al. 1998; Booth \& Wilson 2000). In microscopy, the spherical aberration induced by the refractive index mismatch of the cover glass is often removed by incorporating a correction aberration into the objective lens design. Further benefit is obtained through the use of water immersion objective for imaging aqueous specimens. Some of these objective lenses are equipped with adjustable cover glass correction, to enable the use of different cover glass thicknesses, although careful use is required to ensure correct operation (Schwertner et al. 2005).

A more significant problem is presented by the complex aberrations introduced by the optically inhomogeneous structure of biological specimens (figure 1c). Small but measurable variations have been found in direct measurements of the refractive indices of biological materials and organelles (Bolin et al. 1989; Tearney et al. 1995). These variations can lead to significant aberrations when focusing through thick specimens. Characterization of these aberrations is essential for the understanding of the requirements for a microscope adaptive optics system. Schwertner et al. $(2004 a, b)$ used an interferometer to directly measure specimen-induced aberrations and quantified the results in terms of Zernike function coefficients (figure 2). The aberrations can also be inferred if one knows the refractive index profile of the specimen. 

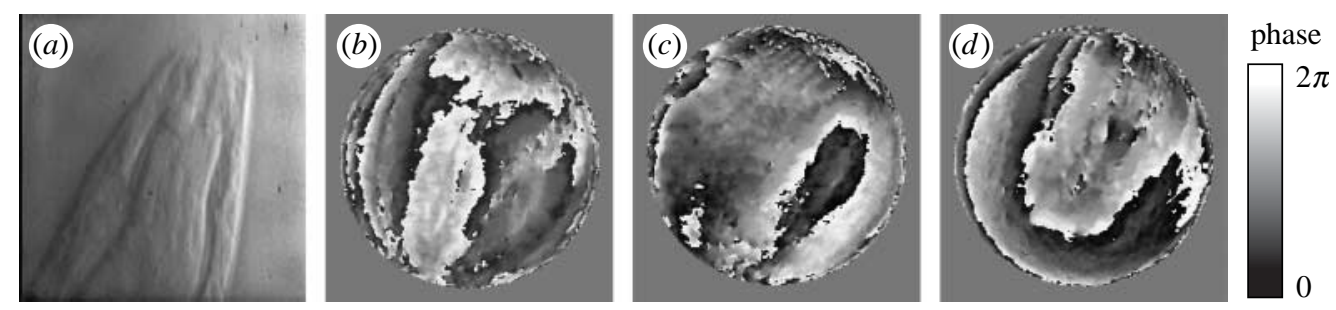

Figure 2. (a) Specimen-induced aberrations from a Caenorhabditis elegans specimen (transmitted light, dimensions $60 \times 60 \mu \mathrm{m})$. $(b-d)$ The three example images show the phase in the objective lens pupil. These phase data are reconstructed from the direct interferometric measurements of $633 \mathrm{~nm}$ wavelength light focused through the specimen using a 1.2 NA water immersion objective (Schwertner et al. 2004b).

Kam et al. (2001) used Normarski differential interference microscopy to reconstruct this profile. Refractive index profiles have also been reconstructed using tomographic holographic microscopy (Charrière et al. 2006).

It should be noted that not all aberrations are caused by the specimen. Although great effort goes into the design of high-resolution microscopes to ensure that they operate at the diffraction limit, aberrations caused by imperfections in the optical system can still be present. For example, if a lens is used off-axis or with the wrong image conjugate, aberrations will be present to some degree (Born \& Wolf 1983; Sheppard \& Gu 1991). They may also arise when the system is not used under design conditions, for example with wrong wavelength or incorrect temperature (Juškaitis 2006).

\section{(c) Effects of aberrations}

There is a complex relationship between the phase aberrations in the pupil and the form of the focal spot. However, broadly speaking, aberrations lead to spreading of the focus both in the lateral plane and, more importantly, an elongation along the optic axis. This is accompanied by a reduction in the focal intensity. The distortion of the focal spot reduces the resolution of the system and causes a blurring between adjacent planes along the optic axis. The drop in intensity leads to a loss of optical efficiency and image contrast. Multiphoton processes are strongly affected by aberrations as the absorption probability is nonlinearly dependent on the focal intensity. In the confocal microscope, the fluorescence generation decreases in proportion to the focal intensity. However, the imaging of this fluorescence onto the detector pinhole is also affected, so the detected signal falls more rapidly due to the compound effect of aberrations in both the illumination and detection paths. Therefore, signal levels in confocal and two-photon microscopes are affected to a similar degree by aberrations (Gu \& Sheppard 1995; Ganic et al. 2000; Booth \& Wilson 2001). Signal levels can be restored by increasing the illumination laser power to compensate for the lower focal intensity, although this does nothing to restore the resolution. It also has the disadvantages of increasing photobleaching and photo-toxic effects - this is of particular importance in live cell imaging, where the lowest exposures are desirable (Hoebe et al. 2007). 


\section{(d) Constructive use of aberrations}

It would be wrong on the part of the reader to think that aberrations are unconditionally detrimental to microscopy. Indeed, there are examples where aberrations have been put to good use in enhancing microscope images. It is possible to adjust imaging properties by introducing a phase pattern into the lens pupil (Neil et al. 2000c). This effect is most beneficial when combined with nonlinear methods, such as stimulated emission depletion (STED) microscopy (Hell 2007). In another method known as wavefront coding, aberrations are intentionally introduced in order to extend the depth of the focus of microscopes (Tucker et al. 1999). When imaging thick specimens, it is possible that light from out-of-focus planes contributes a significant background signal. While aberrations strongly affect in-focus image quality, they have little effect on out-of-focus background. This observation has allowed Leray \& Mertz (2006) to remove the background fluorescence in multiphoton microscopy by subtracting a heavily aberrated image from an aberration-free image.

\section{Adaptive optics for microscopy}

\section{(a) Implementations of adaptive optics in microscopy}

One of the earliest implementations of adaptive optics in microscopy was the use of tip/tilt correction in a transmission confocal microscope (O'Byrne et al. 1999). The major problem encountered here is that the position of the spot at the detector is shifted laterally by structures in the specimen. The authors proposed using a tilting mirror in the transmission path to maintain the position of the focal spot on the detector. Unlike this system, most microscopes are used with epi illumination, with illumination and detection light passing through the same objective lens. In this epi configuration, tip/tilt and defocus aberrations are self-correcting, hence the focus is always conjugate to the detector. It should be noted, however, that this phenomenon does lead to image distortion (Schwertner et al. in press).

The confocal microscope provides maximum resolution and signal level when both illumination and emission paths are diffraction limited. As the illumination and fluorescence light both pass through the specimen, aberrations will be introduced into both the paths. It is clear that to restore diffraction-limited operation, aberration correction must be performed in both the paths. This dual path correction is readily implemented using a single deformable mirror (DM), a configuration that has been employed successfully in confocal fluorescence microscopes (Booth et al. 2002b; Wright et al. 2005; figure 3a). As two-photon microscopes are normally used without a detector pinhole, aberrations in the detection path are unimportant as they have no effect on the resolution or signal level. Therefore, an adaptive two-photon microscope needs only incorporate aberration correction in the illumination path. Such systems have been implemented using DMs (Sherman et al. 2002; Marsh et al. 2003; Rueckel et al. 2006) and liquid crystal spatial light modulators (Neil et al. 2000b).

In addition to scanning microscopes, adaptive optics has also been implemented in imaging microscopes. Adaptive correction for the imaging of two-dimensional objects has been demonstrated in a transmission microscope 

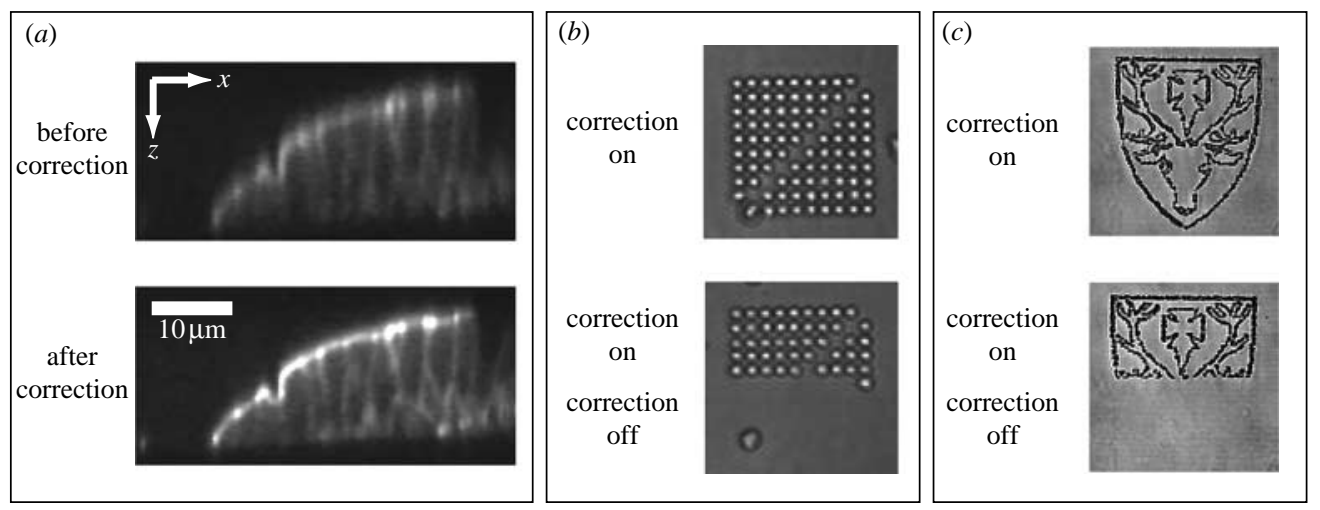

Figure 3. (a) Adaptive confocal fluorescence microscope images of labelled mouse intestine. Aberrations were determined using sequential modal wavefront sensing (Booth et al. 2002b). (b) Transmission microscope images of simulated bit data recorded as voids at a depth of $100 \mu \mathrm{m}$ in a multilayer optical data storage device (Booth et al. 2006b). The exposure for the upper and lower images was identical, except that the aberration correction was switched off for part of the lower pattern. Image size $20 \mu \mathrm{m}$. (c) Aberration correction for optical micro-machining of channels in a polymer substrate (Schwertner et al. 2006). The lower image shows that the machining process is only effective with aberration correction. Image size $80 \mu \mathrm{m}$.

(Débarre et al. 2007). In a different application, predictive aberration correction has been used to extend the field of view of a microscope. Potsaid et al. (2005) combined an objective lens designed to have residual aberrations with a DM that compensated aberrations over a sub-region of the field of view. In this way, they were able to maintain diffraction-limited performance for a sequence of subimages over a much extended field.

\section{(b) Wavefront sensing and aberration measurement}

Several types of wavefront sensing have been developed for adaptive optics, the most prominent methods being the Shack-Hartman wavefront sensor and interferometric sensors (Tyson 1991; Hardy 1998). These methods require a point-like reference source, such as the distant guide star used in astronomical systems, to produce a well-defined wavefront. In adaptive microscopy, the situation is more complicated as the three-dimensional structure of the specimen means that the reference source is generally far from point-like. In effect, the wavefront sensor would receive a multitude of wavefronts, each emanating from different parts of the specimen. Therefore, direct wavefront sensing is not straightforward in microscopy.

Further problems arise if reflected or scattered light is used as the reference source, as the coherent interaction of the light and the specimen structure can cause ambiguity in the wavefront measurement. This effect was discussed by Artal et al. (1995) in the context of ocular imaging. For specular reflection from a planar object, odd (asymmetric) aberrations in the illumination and reflection paths are cancelled, whereas the even (symmetric) components add up. For diffuse scattering, both odd and even aberrations can be measured. This problem does not occur for fluorescence emission, as the phase information from the illumination wavefront is always lost in the incoherent fluorescence process. 
Most of the adaptive microscope systems so far implemented have not employed a wavefront sensor but have used indirect methods of aberration measurement. This approach requires relatively minor modification of the microscope, i.e. the inclusion of an adaptive optical element, and avoids the wavefront sensor pitfalls described previously. In general, this indirect approach involves the maximization of the photodetector signal using an appropriate optimization scheme. Several of these implementations have used stochastic methods based upon genetic algorithms or hill-climbing algorithms (Albert et al. 2000; Sherman et al. 2002; Marsh et al. 2003; Wright et al. 2005). These methods tend to require a large number of iterations that might not be practical for some applications where high speed or low exposure is required. Model-based approaches based upon modal wavefront sensing have shown better performance, requiring fewer measurements than model-free methods (Booth et al. 2002b; Booth 2006, 2007). Stochastic gradient descent algorithms (Vorontsov 2002) have been used in other areas of adaptive optics and might also have useful application in microscopy. Indirect aberration measurement through phase retrieval from images has also been suggested (Hanser et al. 2004).

Direct wavefront sensing can be applicable to microscopy if combined with a method for excluding out-of-focus light. For example, the modal wavefront sensor introduced by Neil et al. employs pinholes that behave much like the detector in a confocal microscope (Neil et al. 2000a; Booth et al. 2002a). Feierabend et al. (2004) have implemented a wavefront sensor that uses coherence gating to exclude the effects of out-of-focus light. This is a promising method for wavefront sensing in thick scattering specimens, such as brain tissue (Rueckel et al. 2006). However, in both cases, measurements are still to a degree dependent upon the specimen structure.

\section{(c) Aberration correction devices}

The commercial availability of practical, affordable correction devices has probably been the main driving factor in adaptive optics for microscopy. The development of liquid crystal (LC) panels for displays has led to spatial light modulators for research applications. These have been demonstrated in adaptive microscopy (Neil et al. 2000b), but have not been widely applied. The main drawbacks of LC devices are their polarization and wavelength dependence, which are not compatible with fluorescence microscopy. LC devices have, however, found wider application in optical data storage and optical trapping (see §4).

Microelectrical mechanical systems (MEMS) technology has enabled the production of a number of DM systems. These are particularly useful for fluorescence imaging, where the high optical efficiency of the mirror ensures that optical losses are low. The most widely used DMs have continuous reflective surfaces, although segmented MEMS mirrors are also becoming available (Dagel et al. 2006). Electrostatically actuated membrane DMs consist of a thin membrane with a reflective coating mounted above an electrode array (Vdovin et al. 1999). By applying a potential between the membrane and the electrode, the DM surface shape can be pulled towards the electrodes. A transparent electrode can also be included above the membrane to permit push-pull deformations (Bonora \& Poletto 2006). Other electrostatically actuated MEMS DMs have been based upon microstructured silicon (Bifano et al. 1999). Here a supporting structure below 
the reflective membrane ensures that the influence of each actuator on the DM shape is spatially more confined than for a membrane DM. Piezo-electric actuators have been employed in DMs both using direct actuation of the mirror surface (Simonov et al. 2006) and through curvature control using bimorph actuators (Dainty et al. 1998). Magnetically actuated DMs have also been demonstrated (Fernandez et al. 2006). These DMs have been complemented by more exotic technologies such as thermally actuated (Vdovin \& Loktev 2002), fluidic (Vuelban et al. 2006) and ferromagnetic devices (Laird et al. 2006).

Given the wide range of DMs available, the selection of an appropriate device for a particular application is a non-trivial task. While properties such as cost, size, speed and robustness are relatively self-explanatory, the aberration correction ability of a device is harder to evaluate. The standard quantities specified by manufacturers, such as maximum stroke or number of actuators, are not themselves useful guides to the compatibility of a device with an aberration correction task-more is not necessarily better. Several methods have been developed to characterize DMs and determine the range of correctable aberration modes (Zhu et al. 1999; Fernandez \& Artal 2003; Booth et al. 2005) and the performance for particular correction tasks (Paterson et al. 2000; Dalimier \& Dainty 2005).

\section{Other applications of adaptive optics}

The optical systems used in high-resolution microscopy are also central to other applications in optical and photonic engineering. These include data storage, optical trapping and micro/nanofabrication. All of these applications require the resolution provided by the tight focusing of light using high-NA objective lenses. As in microscopy, these methods suffer from aberrations introduced as the focused light passes through different parts of the specimen or substrate. The efficiency and accuracy of all of these processes are detrimentally affected by aberrations and the use of adaptive optics has great potential.

Much of the recent research into three-dimensional optical data storage has used multiphoton processes to record data and confocal microscopes for read-out. As these systems invariably involve focusing through a refractive index mismatch, aberration control is an important consideration in system design and the use of adaptive aberration correction has been explored. In these systems, the recording substrate is predominantly isotropic, so aberrations can be predicted from the focusing depth, obviating the need for direct aberration measurement. LC devices have been used in single or dual recording layer systems to correct spherical aberration due to the disc thickness and coma caused by disc tilt (Ohtaki et al. 1999; Somalingam et al. 2004; Knittel et al. 2005). The aberrations become more significant in three-dimensional storage where focusing depths are much larger. Early work in three-dimensional memory involved the compensation of spherical aberration by adjusting the tube length of the objective lens (Day \& Gu 1998). Neil et al. (2002) employed an LC device to record data at a depth of $1 \mathrm{~mm}$ in a high refractive index medium. Booth et al. (2006) have demonstrated a DM-based system for aberration correction in the recording and read-out of multilayer polymer media (figure $3 b$ ). 
Optical tweezers have been widely applied for the manipulation of small particles on the micrometre scale (Grier 2003; McGloin 2006). The effectiveness of optical tweezers is compromised by aberrations (Rohrbach \& Stelzer 2002; Ganic et al. 2004; Roichman et al. 2006; Vermeulen et al. 2006) and adaptive optics have been applied by various groups to improve performance. Tube length correction was employed by Ke \& Gu (1998) to compensate for refractive index mismatch-induced aberrations. Ota et al. (2003) used a DM to manually adjust spherical aberration introduced when trapping in an aqueous medium using an oil immersion objective. Theofanidou et al. (2004) optimized the two-photon fluorescence signal from a trapped fluorescent bead to increase trap strength. Aberrations have also been compensated in a system employing a LC spatial light modulator as a holographic element (Wulff et al. 2006).

Optical methods of nanofabrication have been used to manufacture polymer, ceramic and metallic structures with feature sizes as small as $100 \mathrm{~nm}$ (Serbin et al. 2003; Sun \& Kawata 2004; Ishikawa et al. 2006). This sub-wavelength resolution is achieved through the use of nonlinear, thresholded optical processes. Thresholded multiphoton processes have also been employed to machine structures in high refractive index media for the manufacture of photonic crystals (Wong et al. 2006; Zhou \& Gu 2006). As these methods require focusing between materials of different refractive indices, they suffer from aberrations that can reduce the focal intensity. If this drops below the threshold level, the fabrication effect can be lost entirely. Adaptive optics can be used to maintain focal spot quality even when focusing at depth in the fabrication substrate (Schwertner et al. 2006; figure 3c).

\section{Future outlook}

The principles of adaptive optics for microscopy and photonic engineering have been demonstrated in a number of implementations and applications. However, there are still many technical challenges to be met before adaptive optics becomes a standard feature. Studies have shown that specimen-induced aberrations can change significantly over a microscope's field of view (Schwertner et al. 2004a,b), so real-time adaptive optics for confocal and twophoton microscopes would be desirable. This may only be possible with improved aberration measurement schemes and faster correction devices - current DMs are limited in speed to a few kilohertz, whereas the fastest pixel rates in commercial microscopes can be in excess of $100 \mathrm{kHz}$. Advances could be made in correcting field-dependent aberrations through the use of multiconjugate adaptive optics, where multiple adaptive elements are employed (Kam et al. 2007). This would also enable the use of adaptive optics in widefield microscopes, including the widefield sectioning microscopes that produce confocal-like images (Conchello \& Lichtman 2005). There is also much scope for the use of adaptive optics in emerging microscopy methods. This is especially the case in microscopies that rely upon nonlinear effects, such as third harmonic generation (Débarre et al. 2006), coherent anti-Stokes Raman microscopy (Zumbusch et al. 1999) or STED microscopy (Hell 2007). Perhaps one of the major benefits of microscope adaptive optics will be for in vivo tissue 
imaging, as aberration correction provides better image contrast with the use of lower illumination intensities, correspondingly lower phototoxicity and improved specimen viability.

In three-dimensional optical data storage, the predominant challenge is the production of compact, low-cost and robust adaptive devices that can be incorporated into an optical drive. The most promising candidates seem to be LC devices that are currently being used in optical disc systems (Knittel et al. 2005). Most of the current work in aberration correction for optical tweezers has concerned the compensation of low-order spherical aberration. For trapping within complex biological specimens, it will be necessary to develop more advanced adaptive schemes. Optical fabrication methods will be more affected by aberrations as the size and complexity of the fabricated structures are increased. Again advances in microscope adaptive optics will help maintain the precision of the fabrication systems.

Images in figures 2 and 3 are reproduced with the kind permission of M. Schwertner and T. Wilson. The author is grateful to E. Botcherby, D. Débarre, R. Juškaitis, M. Schwertner and T. Wilson for numerous useful discussions concerning this material.

\section{References}

Albert, O., Sherman, L., Mourou, G., Norris, T. B. \& Vdovin, G. 2000 Smart microscope: an adaptive optics learning system for aberration correction in multiphoton confocal microscopy. Opt. Lett. 25, 52-54. (doi:10.1364/OL.25.000052)

Artal, P., Marcos, S., Navarro, R. \& Williams, D. R. 1995 Odd aberrations and double-pass measurements of retinal image quality. J. Opt. Soc. Am. A 12, 195-201.

Bifano, T. G., Perreault, J., Krishnamoorthy, R. \& Horenstein, M. N. 1999 Microelectromechanical deformable mirrors. IEEE J. Sel. Topics Quantum Electron. 5, 83-89. (doi:10.1109/2944.748109)

Bolin, F. P., Preuss, L. E., Taylor, R. C. \& Ference, R. J. 1989 Refractive index of some mammalian tissues using a fiber optic cladding method. Appl. Opt. 28, 2279-2303.

Bonora, S. \& Poletto, L. 2006 Push-pull membrane mirrors for adaptive optics. Opt. Express 14, 11 935-11 944. (doi:10.1364/OE.14.011935)

Booth, M. J. 2006 Wave front sensor-less adaptive optics: a model-based approach using sphere packings. Opt. Express 14, 1339-1352. (doi:10.1364/OE.14.001339)

Booth, M. J. 2007 Wavefront sensorless adaptive optics for large aberrations. Opt. Lett. 32, 5-7. (doi:10.1364/OL.32.000005)

Booth, M. J. \& Wilson, T. 2000 Strategies for the compensation of specimen induced aberration in confocal microscopy of skin. J. Microsc. 200, 68-74. (doi:10.1046/j.1365-2818.2000.00735.x)

Booth, M. J. \& Wilson, T. 2001 Refractive-index-mismatch induced aberrations in single-photon and two-photon microscopy and the use of aberration correction. J. Biomed. Opt. 6, 266-272. (doi:10.1117/1.1382808)

Booth, M. J., Neil, M. A. A. \& Wilson, T. 1998 Aberration correction for confocal imaging in refractive-index-mismatched media. J. Microsc. 192, 90-98. (doi:10.1111/j.1365-2818.1998. 99999.x)

Booth, M. J., Neil, M. A. A. \& Wilson, T. $2002 a$ New modal wave-front sensor: application to adaptive confocal fluorescence microscopy and two-photon excitation fluorescence microscopy. J. Opt. Soc. Am. A 19, 2112-2120. (doi:10.1364/JOSAA.19.002112)

Booth, M. J., Neil, M. A. A., Juškaitis, R. \& Wilson, T. $2002 b$ Adaptive aberration correction in a confocal microscope. Proc. Natl Acad. Sci. USA 99, 5788-5792. (doi:10.1073/pnas.082544799)

Booth, M. J., Wilson, T., Sun, H.-B., Ota, T. \& Kawata, S. 2005 Methods for the characterisation of deformable membrane mirrors. Appl. Opt. 44, 5131-5139. (doi:10.1364/AO.44.005131) 
Booth, M. J., Schwertner, M., Wilson, T., Nakano, M., Kawata, Y., Nakabayashi, M. \& Miyata, S. 2006 Predictive aberration correction for multilayer optical data storage. Appl. Phys. Lett. 88, 031 109. (doi:10.1063/1.2166684)

Born, M. \& Wolf, E. 1983 Principles of optics, 6th edn. Oxford, UK: Pergamon Press.

Charrière, F., Marian, A., Montfort, F., Kuehn, J., Colomb, T., Cuche, E., Marquet, P. \& Depeursinge, C. 2006 Cell refractive index tomography by digital holographic microscopy. Opt. Lett. 31, 178-180. (doi:10.1364/OL.31.000178)

Conchello, J. A. \& Lichtman, J. W. 2005 Optical sectioning microscopy. Nat. Methods 2, 920-931. (doi:10.1038/nmeth815)

Dagel, D. J. et al. 2006 Large-stroke mems deformable mirrors for adaptive optics. J. Microelectromech. Syst. 15, 572-583. (doi:10.1109/JMEMS.2006.876794)

Dainty, J. C., Koryabin, A. V. \& Kudryashov, A. V. 1998 Low-order adaptive deformable mirror. Appl. Opt. 37, 4663-4668.

Dalimier, E. \& Dainty, J. C. 2005 Comparative analysis of deformable mirrors for ocular adaptive optics. Opt. Express 13, 4275-4285. (doi:10.1364/OPEX.13.004275)

Day, D. \& Gu, M. 1998 Effects of refractive-index mismatch on three-dimensional optical datastorage density in a two-photon bleaching polymer. Appl. Opt. 37, 6299-6304.

Débarre, D., Supatto, W., Pena, A.-M., Fabre, A., Tordjmann, T., Combettes, L., Schanne-Klein, M.-C. \& Beaurepaire, E. 2006 Imaging lipid bodies in cells and tissues using third-harmonic generation microscopy. Nat. Methods 3, 47-53. (doi:10.1038/nmeth813)

Débarre, D., Booth, M. J. \& Wilson, T. 2007 Image-based adaptive optics through the optimisation of low spatial frequencies. Opt. Express 15, 8176-8190. (doi:10.1364/OE.15.008176)

Egner, A. \& Hell, S. W. 1998 Equivalence of the Huygens-Fresnel and Debye approach for the calculation of high aperture point-spread functions in the presence of refractive index mismatch. J. Microsc. 193, 244-249. (doi:10.1046/j.1365-2818.1999.00462.x)

Feierabend, M., Rückel, M. \& Denk, W. 2004 Coherence-gated wave-front sensing in strongly scattering samples. Opt. Lett. 29, 2255-2257. (doi:10.1364/OL.29.002255)

Fernandez, E. J. \& Artal, P. 2003 Membrane deformable mirror for adaptive optics: performance limits in visual optics. Opt. Express 11, 1056-1069.

Fernandez, E. J., Vabre, L., Hermann, B., Unterhuber, A., Povazay, B. \& Drexler, W. 2006 Adaptive optics with a magnetic deformable mirror: applications in the human eye. Opt. Express 14, 8900-8917. (doi:10.1364/OE.14.008900)

Ganic, D., Gan, X. S. \& Gu, M. 2000 Reduced effects of spherical aberration on penetration depth under two-photon excitation. Appl. Opt. 39, 3945-3947.

Ganic, D., Gan, X. S. \& Gu, M. 2004 Exact radiation trapping force calculation based on vectorial diffraction theory. Opt. Express 12, 2670-2675. (doi:10.1364/OPEX.12.002670)

Grier, D. G. 2003 A revolution in optical manipulation. Nature 424, 810-816. (doi:10.1038/ nature01935)

Gu, M. 1996 Principles of three-dimensional imaging in confocal microscopes. Singapore: World Scientific.

Gu, M. \& Sheppard, C. J. R. 1995 Comparison of 3D imaging properties between 2-photon and single-photon fluorescence microscopy. J. Microsc. 177, 128-137.

Hanser, B. M., Gustafsson, M. G. L., Agard, D. A. \& Sedat, J. W. 2004 Phase-retrieved pupil functions in wide-field fluorescence microscopy. J. Microsc. 216, 36-48. (doi:10.1111/j.0022-2720.2004.01393.x)

Hardy, J. W. 1998 Adaptive optics for astronomical telescopes. Oxford, UK: Oxford University Press.

Hell, S. W. 2007 Far-field optical nanoscopy. Science 316, 1153-1158. (doi:10.1126/science.1137395)

Hoebe, R. A., Van Oven, C. H., Gadella, T. W. J., Dhonukshe, P. B., Van Noorden, C. J. F. \& Manders, E. M. M. 2007 Controlled light-exposure microscopy reduces photobleaching and phototoxicity in fluorescence live-cell imaging. Nat. Biotechnol. 25, 249-253. (doi:10.1038/nbt1278)

Ishikawa, A., Tanaka, T. \& Kawata, S. 2006 Improvement in the reduction of silver ions in aqueous solution using two-photon sensitive dye. Appl. Phys. Lett. 89, 113 102. (doi:10.1063/1.2345601) 
Juškaitis R. 2006 Measuring the real point spread function of high numerical aperture microscope objective lenses. In Handbook of biological confocal microscopy (ed. J. B. Pawley), pp. 239-250, 3rd edn. New York, NY: Springer.

Kam, Z., Hanser, B., Gustafsson, M. G., Agard, D. A. \& Sedat, J. W. 2001 Computational adaptive optics for live three-dimensional biological imaging. Proc. Natl Acad. Sci. USA 98, 3790-3795. (doi:10.1073/pnas.071275698)

Kam, Z., Kner, P., Agard, D. \& Sedat, J. W. 2007 Modelling the application of adaptive optics to wide-field microscope live imaging. J. Microsc. 226, 33-42.

Ke, P. C. \& Gu, M. 1998 Characterization of trapping force in the presence of spherical aberration. J. Modern Opt. 45, 2159-2168.

Knittel, J., Richter, H., Hain, M., Somalingam, S. \& Tschudi, T. 2005 Liquid crystal lens for spherical aberration compensation in a blu-ray disc system. IEE Proc. Sci. Measure. Technol. 152, 15-18. (doi:10.1049/ip-smt:20051044)

Laird, P., Caron, N., Rioux, M., Borra, E. F. \& Ritcey, A. 2006 Ferrofluidic adaptive mirrors. Appl. Opt. 45, 3495-3500. (doi:10.1364/AO.45.003495)

Leray, A. \& Mertz, J. 2006 Rejection of two-photon fluorescence background in thick tissue by differential aberration imaging. Opt. Express 14, 10 565-10 573. (doi:10.1364/OE.14.010565)

Mahajan, V. N. 2001 Optical imaging and aberrations, part II. Wave diffraction optics. Bellingham, WA: SPIE.

Marsh, P. N., Burns, D. \& Girkin, J. M. 2003 Practical implementation of adaptive optics in multiphoton microscopy. Opt. Express 11, 1123-1130.

McGloin, D. 2006 Optical tweezers: 20 years on. Phil. Trans. R. Soc. A 17, 3521-3537. (doi:10. 1098/rsta.2006.1891)

Neil, M. A. A., Booth, M. J. \& Wilson, T. $2000 a$ New modal wavefront sensor: a theoretical analysis. J. Opt. Soc. Am. A 17, 1098-1107. (doi:10.1364/JOSAA.17.001098)

Neil, M. A. A., Juškaitis, R., Booth, M. J., Wilson, T., Tanaka, T. \& Kawata, S. $2000 b$ Adaptive aberration correction in a two-photon microscope. J. Microsc. 200, 105-108. (doi:10.1046/ j.1365-2818.2000.00770.x)

Neil, M. A. A., Wilson, T. \& Juškaitis, R. 2000c A wavefront generator for complex pupil function synthesis and point spread function engineering. J. Microsc. 197, 219-223. (doi:10.1046/j.13652818.2000.00680.x)

Neil, M. A. A., Juškaitis, R., Booth, M. J., Wilson, T., Tanaka, T. \& Kawata, S. 2002 Active aberration correction for the writing of three-dimensional optical memory devices. Appl. Opt. 41, 1374-1379. (doi:10.1364/AO.41.001374)

O’Byrne, J. W., Fekete, P. W., Arnison, M. R., Serrano, M., Zhao, H., Philp, D., Sudiarta, W., Cogswell, C. J. 1999 Adaptive optics in confocal microscopy. In Proc. 2nd Int. Workshop on Adaptive Optics for Industry and Medicine. Singapore: World Scientific.

Ohtaki, S., Murao, N., Ogasawara, M. \& Iwasaki, M. 1999 The applications of a liquid crystal panel for 15 GB optical disk systems. Jpn J. Appl. Phys. 38, 1744-1749. (doi:10.1143/JJAP.38. 1744)

Ota, T., Sugiura, T., Kawata, S., Booth, M. J., Neil, M. A. A., Juskaitis, R. \& Wilson, T. 2003 Enhancement of laser trapping force by spherical aberration correction using a deformable mirror. Jpn J. Appl. Phys. 42, L701-L703. (doi:10.1143/JJAP.42.L701)

Paterson, C., Munro, I. \& Dainty, J. C. 2000 A low cost adaptive optics system using a membrane mirror. Opt. Express 6, 175-185.

Pawley, J. B. 2006 Handbook of biological confocal microscopy, 3rd edn. New York, NY: Springer.

Potsaid, B., Bellouard, Y. \& Wen, J. T. 2005 Adaptive scanning optical microscope (ASOM): a multidisciplinary optical microscope design for large field of view and high resolution imaging. Opt. Express 13, 6504-6518. (doi:10.1364/OPEX.13.006504)

Rohrbach, A. \& Stelzer, E. H. K. 2002 Trapping forces, force constants, and potential depths for dielectric spheres in the presence of spherical aberrations. Appl. Opt. 41, 2494-2507. (doi:10. 1364/AO.41.002494) 
Roichman, Y., Waldron, A., Gardel, E. \& Grier, D. G. 2006 Optical traps with geometric aberrations. Appl. Opt. 45, 3425-3429. (doi:10.1364/AO.45.003425)

Rueckel, M., Mack-Bucher, J. A. \& Denk, W. 2006 Adaptive wavefront correction in two-photon microscopy using coherence-gated wavefront sensing. Proc. Natl Acad. Sci. USA 103, 17 137-17 142. (doi:10.1073/pnas.0604791103)

Schwertner, M., Booth, M. J., Neil, M. A. A. \& Wilson, T. $2004 a$ Measurement of specimeninduced aberrations of biological samples using phase stepping interferometry. J. Microsc. 213, 11-19. (doi:10.1111/j.1365-2818.2004.01267.x)

Schwertner, M., Booth, M. J. \& Wilson, T. $2004 b$ Characterizing specimen induced aberrations for high NA adaptive optical microscopy. Opt. Express 12, 6540-6552. (doi:10.1364/OPEX.12.006540)

Schwertner, M., Booth, M. J. \& Wilson, T. 2005 Simple optimization procedure for objective lens correction collar setting. J. Microsc. 217, 184-187. (doi:10.1111/j.1365-2818.2005.01431.x)

Schwertner, M., Booth, M. J. \& Wilson, T. 2006 Adaptive optics for microscopy, optical data storage, and micromachining. Proc. SPIE 6306, 63060A. (doi:10.1117/12.681036)

Schwertner, M., Booth, M. J. \& Wilson, T. In press. Specimen-induced distortions in light microscopy. J. Microsc.

Serbin, J. et al. 2003 Femtosecond laser-induced two-photon polymerization of inorganic-organic hybrid materials for applications in photonics. Opt. Lett. 28, 301-303. (doi:10.1364/OL.28. 000301)

Sheppard, C. J. R. \& Cogswell, C. J. 1991 Effects of aberrating layers and tube length on confocal imaging properties. Optik 87, 34-38.

Sheppard, C. J. R. \& Gu, M. 1990 Image-formation in 2-photon fluorescence microscopy. Optik 86, 104-106.

Sheppard, C. J. R. \& Gu, M. 1991 Aberration compensation in confocal microscopy. Appl. Opt. 30, 3563-3568.

Sherman, L., Ye, J. Y., Albert, O. \& Norris, T. B. 2002 Adaptive correction of depth-induced aberrations in multiphoton scanning microscopy using a deformable mirror. J. Microsc. 206, 65-71. (doi:10.1046/j.1365-2818.2002.01004.x)

Simonov, A. N., Hong, S. \& Vdovin, G. 2006 Piezoelectric deformable mirror with adaptive multiplexing control. Opt. Eng. 45, 070501. (doi:10.1117/1.2219733)

Somalingam, S., Dressbach, K., Hain, M., Stankovic, S., Tschudi, T., Knittel, J. \& Richter, H. 2004 Effective spherical aberration compensation by use of a nematic liquid-crystal device. Appl. Opt. 43, 2722-2729. (doi:10.1364/AO.43.002722)

Sun, H. B. \& Kawata, S. 2004 Two-photon photopolymerization and 3D lithographic microfabrication. NMR 3D Anal. Photopolym. 170, 169-273.

Tearney, G. J., Brezinski, M. E., Southern, J. F., Bouma, B. E., Hee, M. R. \& Fujimoto, J. G. 1995 Determination of the refractive index of highly scattering human tissue by optical coherence tomography. Opt. Lett. 20, 2258-2260.

Theofanidou, E., Wilson, L., Hossack, W. J. \& Arlt, J. 2004 Spherical aberration correction for optical tweezers. Opt. Commun. 236, 145-150. (doi:10.1016/j.optcom.2004.03.009)

Török, P., Varga, P. \& Németh, G. 1995 Analytic solution of the diffraction integrals and interpretation of wave-front distortion when light is focussed through a planar interface between materials of mismatched refractive indices. J. Opt. Soc. Am. A 12, 2660-2671.

Tucker, S. C., Cathey, W. T. \& Dowski, E. R. 1999 Extended depth of field and aberration control for inexpensive digital microscope systems. Opt. Express 4, 467-474.

Tyson, R. K. 1991 Principles of adaptive optics. London, UK: Academic Press.

Vdovin, G. \& Loktev, M. 2002 Deformable mirror with thermal actuators. Opt. Lett. 27, 677-679. (doi:10.1364/OL.27.000677)

Vdovin, G., Sarro, P. M. \& Middelhoek, S. 1999 Technology and applications of micromachined adaptive mirrors. J. Micromech. Microeng. 9, R8-R20. (doi:10.1088/0960-1317/9/2/202)

Vermeulen, K. C., Wuite, G. J. L., Stienen, G. J. M. \& Schmidt, C. F. 2006 Optical trap stiffness in the presence and absence of spherical aberrations. Appl. Opt. 45, 1812-1819. (doi:10.1364/AO. 45.001812 ) 
Vorontsov, M. A. 2002 Decoupled stochastic parallel gradient descent optimization for adaptive optics: integrated approach for wave-front sensor information fusion. J. Opt. Soc. Am. A 19, 356-368. (doi:10.1364/JOSAA.19.000356)

Vuelban, E. M., Bhattacharya, N. \& Braat, J. J. M. 2006 Liquid deformable mirror for high-order wavefront correction. Opt. Lett. 31, 1717-1719. (doi:10.1364/OL.31.001717)

Wilson, T. 1990 Confocal microscopy. London, UK: Academic Press.

Wong, S., Deubel, M., Perez-Willard, F., John, S., Ozin, G. A., Wegener, M. \& von Freymann, G. 2006 Direct laser writing of three-dimensional photonic crystals with a complete photonic bandgap in chalcogenide glasses. Adv. Mater. 18, 265-269. (doi:10.1002/adma.200501973)

Wright, A. J., Burns, D., Patterson, B. A., Poland, S. P., Valentine, G. J. \& Girkin, J. M. 2005 Exploration of the optimisation algorithms used in the implementation of adaptive optics in confocal and multiphoton microscopy. Microsc. Res. Tech. 67, 36-44. (doi:10.1002/jemt.20178)

Wulff, K. D., Cole, D. G., Clark, R. L., DiLeonardo, R., Leach, J., Cooper, J., Gibson, G. \& Padgett, M. J. 2006 Aberration correction in holographic optical tweezers. Opt. Express 14, 4169-4174. (doi:10.1364/OE.14.004169)

Zernike, F. 1934 Beugungstheorie des Schneidenverfahrens und seiner verbesserten Form, der Phasenkontrastmethode. Physica (Utrecht) 1, 689-704. (doi:10.1016/S0031-8914(34)80259-5)

Zhou, G. Y. \& Gu, M. 2006 Direct optical fabrication of three-dimensional photonic crystals in a high refractive index $\mathrm{LiNbO}_{3}$ crystal. Opt. Lett. 31, 2783-2785. (doi:10.1364/OL.31.002783)

Zhu, L. J., Sun, P. C., Bartsch, D. U., Freeman, W. R. \& Fainman, Y. 1999 Wave-front generation of Zernike polynomial modes with a micromachined membrane deformable mirror. Appl. Opt. 38, 6019-6026.

Zumbusch, A., Holtom, G. R. \& Xie, X. S. 1999 Three-dimensional vibrational imaging by coherent anti-Stokes Raman scattering. Phys. Rev. Lett. 82, 4142-4145. (doi:10.1103/ PhysRevLett.82.4142) 


\title{
AUTHOR PROFILE
}

\author{
Martin J. Booth
}

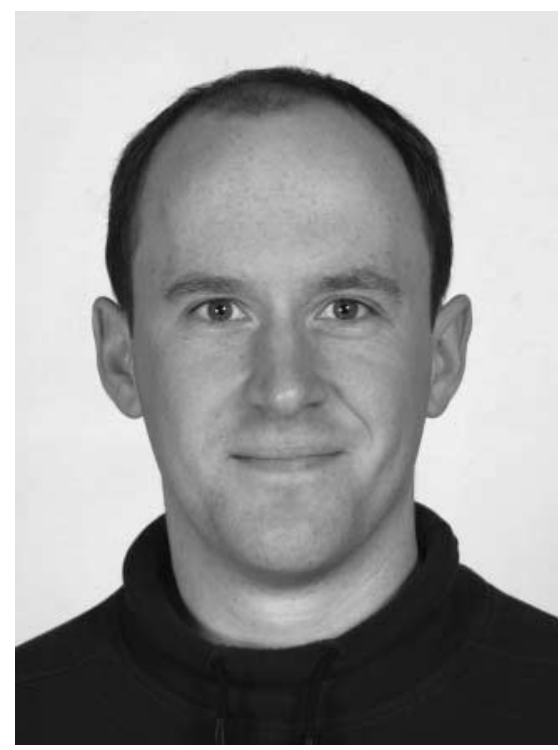

Martin Booth is a Royal Academy of Engineering/EPSRC research fellow in the Department of Engineering Science at the University of Oxford. He received a masters degree (MEng) from Oxford in 1997. This was followed by a research visit to the Max Planck Institute for Biophysical Chemistry in Göttingen, Germany, where he investigated two-photon microscopy using continuous wave illumination. He returned to Oxford to pursue doctoral research into the application of adaptive optics to confocal microscopy. This led to the award of a doctorate (DPhil) in 2001. The same year he was appointed as a junior research fellow of Christ Church, Oxford and he took up his present research fellowship in 2003. His current research interests include the further development of adaptive optics for microscopy and the application of adaptive optics in other areas of optical engineering and photonics. This has included the use of aberration correction for optical data storage, micro/nanofabrication and optical trapping. This work has been centred in Oxford but has also taken place in collaboration with Osaka University, Shizuoka University and RIKEN, Japan, and Swinburne University of Technology in Melbourne, Australia. 